methods with relatively poor sensitivity compared to nucleic acid amplification methods. Our aim was to determine TV prevalence using the APTIMA TV Assay (ATV, Gen-Probe Incorporated) and the frequency of co-infections with Chlamydia trachomatis (CT) and Neisseria gonorrhoea (NG) in the USA among women being screened. Methods Samples from 7593 women aged 18-89 years undergoing routine CT and NG screening at obstetrics/gynaecology, emergency room, hospital in-patient, family practice, family planning, internal medicine, jail, and STD clinic populations in 21 states were collected. Consecutive samples previously tested for CT and NG by the APTIMA COMBO 2 Assay (Gen-Probe Incorporated) were retrospectively tested with the ATV assay. Endocervical, urine, vaginal swab and PreservCyt liquid Pap samples (Hologic Inc.) diluted into APTIMA specimen transport buffer were tested.

Results Overall prevalences of TV, CT and NG in surveyed women were $8.7 \%, 6.7 \%$, and $1.7 \%$, respectively. TV prevalence ranged from 7.5 to $8.6 \%$ in women age 18 to $39 \mathrm{yr}$, and increased to $9.8 \%$ in women age $40-44 \mathrm{yr}$. Highest observed TV prevalences were in women ages $45-49 \mathrm{yr}(13.4 \%)$ and over $50 \mathrm{yr}$ (13.0\%). CT and NG prevalences were less than $2 \%$ in the $40+$ age group and highest in women less than 30 years of age ranging from $5.2 \%$ to $14.3 \%$ for CT and $1.3 \%-3.3 \%$ for NG. TV was the more prevalent STD than either CT or NG in all age groups, except the 18-19 yr group (CT: $14.3 \%$; TV: $8.5 \%)$. TV prevalence differed by race/ethnicity $(20.2 \%$ blacks; $5.7 \%$ whites; $5.0 \%$ Hispanics; $3.8 \%$ Asians). TV prevalence was $14.4 \%$ in the Southeast, $9.5 \%$ in the Southwest and Midwest, and $4.3 \%$ in the Northeast and ranged from $5.4 \%$ in Family Planning clinics to $22.3 \%$ in jails. Co-infections in most age groups were $<1 \%$, and were highest in the 18-19yr group (TV/CT: $2.1 \%$; TV/NG: $0.88 \%)$

Conclusions TV prevalence was highest in women over 40 years of age, in contrast to CT and NG prevalences which were highest in women under 30 years of age. Co-infection of TV with CT or NG was relatively low. The high TV prevalence in all age groups suggests that all women being screened for CT/NG should also be screened for TV. Routine TV screening should also be considered for at-risk sexually active women of any age.

\section{3-S2.06 THE EFFECT OF TRICHOMONAS VAGINALIS (TV) INFECTION ON THE VAGINAL MICROBIOME}

doi:10.1136/sextrans-2011-050109.114

${ }^{1} \mathrm{D}$ H Martin, ${ }^{2} \mathrm{M}$ Zozaya-Hinchliffe, ${ }^{1} \mathrm{R}$ Lillis, ${ }^{1} \mathrm{~J}$ Miller, ${ }^{2} \mathrm{M}$ Ferris. ${ }^{1}$ LSU Health Sciences Center, New Orleans, USA; ${ }^{2}$ Research Institute for Children, New Orleans, USA

Background Among pregnant women the prevalence of TV infection is highest in women who have intermediate Nugent scores (NS) compared to those with low and high scores reflective of normal and bacterial vaginosis (BV) types of vaginal flora. The goals of this study were to determine if this relationship held true for non pregnant women and to determine if TV infection was associated with changes in the vaginal microbiome.

Methods The study subjects were 394 women enrolled in a crosssectional study of the aetiology of cervicitis in the New Orleans STD clinic. TV was diagnosed using the InPouch culture method. NS was determined using standard criteria. C trachomatis (CT), $N$ gonorrhoeae (NG), and $M$ genitalium (MG) were diagnosed using NAATs. DNA was extracted from a vaginal swab and stored. Associations between NS and STIs and NS and sexual behaviour were analysed for all 394 women. 454 pyrosequencing analyses were performed on vaginal DNA from 30 TV positive and 30 TV negative samples evenly divided between those with normal, intermediate, and BV flora as determined by NS.

Results $95 \%$ of enrolled women were African American and the mean age of the population was 25.6 years. The prevalence of TV (y axis) by 5 NS categories ( $x$ axis) is shown in the Abstract O3S2.06 figure 1. As opposed to TV, the prevalence rates for CT, GC, and MG were highest among women with NS of 7-10. Also there was no difference in high risk sexual behaviour between those with low and high NS. These data suggested that the striking decrease in TV prevalence observed among women with BV was the result of vaginal environmental factors, not decreased risk for STIs. A heat map based on pyrosequencing data showed that the vaginal flora of 18/30 of the women positive for TV had similar microbiomes which were distinctly different from those of the other 42 women. In the former group, Mycoplasma spp. and Ureaplasma spp. were more abundant than in the latter group while the reverse was true of Megasphaera spp. and Gardnerella spp.

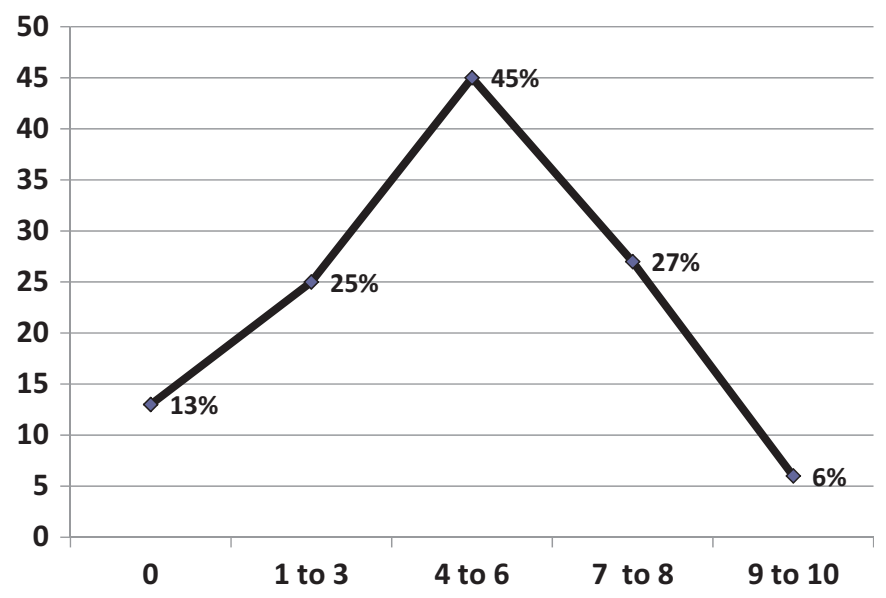

Abstract 03-S2.06 Figure 1 T. vaginalis Prevalence by Nugent Score.

Conclusions Though published research shows that the incidence of TV is highest in women with BV, our data along with those from a previous study in pregnant women clearly establish that TV prevalence is highest in women with intermediate NS. These data in conjunction with our pyrosequencing results suggest that following infection TV modifies the vaginal microbiome by suppressing some of the BV associated organisms and enhancing the abundance of mycoplasmas.

\section{Clinical sciences oral session 3-diagnostic testing: chlamydia \& gonorrhoeae 03-S3.01 COMPARISON OF A NEW APTIMA SPECIMEN COLLECTION AND TRANSPORTATION KIT TO L-PAP FOR DETECTION OF C TRACHOMATIS, N GONORRHOEAE AND T VAGINALIS IN CERVICAL AND VAGINAL SPECIMENS}

doi:10.1136/sextrans-2011-050109.115

M Chernesky. McMaster University, Hamilton, Canada

Background Control of sexually transmitted infections in women has focused on screening using cervical, vaginal or urine samples. The objective was to compare a new specimen collection and transportation (SCT) kit to PreservCyt (PC) and SurePath (SP) liquid-based Pap (L-Pap) for cervical sampling and self- and physician-collected vaginal samples.

Methods Women ( $\mathrm{n}=562$ ) attending a gynaecology clinic (GYC) $(n=435)$ or a youth health clinic (YHC) $(n=127)$ signed consent for a physician to collect two L-Pap samples with a Cervex broom, cervical (CSCT) and vaginal (VSCT) samples and a self-collected VSCT. All specimens were tested for Chlamydia trachomatis (CT) and Neisseria gonorrhoeae (NG) by APTIMA Combo 2 (AC2) and 